\title{
Sliding Window Based Feature Extraction and Traffic Clustering for Green Mobile Cyberphysical Systems
}

\author{
Jiao Zhang, ${ }^{1,2}$ Li Zhou, ${ }^{1,3}$ Angran Xiao, ${ }^{4}$ Sai Zeng, ${ }^{5}$ Haitao Zhao, ${ }^{1}$ and Jibo Wei ${ }^{1}$ \\ ${ }^{1}$ College of Electronic Science and Engineering, National University of Defense Technology, Changsha, China \\ ${ }^{2}$ Shenzhen Institutes of Advanced Technology, Chinese Academy of Sciences, Shenzhen, China \\ ${ }^{3}$ Science and Technology on Information Transmission and Dissemination in Communication Networks Laboratory, \\ Shijiazhuang, China \\ ${ }^{4}$ Department of Mechanical Engineering Technology, New York City College of Technology, City University of New York, \\ Brooklyn, NY 11201, USA \\ ${ }^{5}$ IBM Thomas J. Watson Research Center, Yorktown Heights, NY 10598, USA
}

Correspondence should be addressed to Li Zhou; zhouli2035@nudt.edu.cn

Received 16 February 2017; Accepted 4 May 2017; Published 30 May 2017

Academic Editor: Jun Cheng

Copyright (c) 2017 Jiao Zhang et al. This is an open access article distributed under the Creative Commons Attribution License, which permits unrestricted use, distribution, and reproduction in any medium, provided the original work is properly cited.

\begin{abstract}
Both the densification of small base stations and the diversity of user activities bring huge challenges for today's heterogeneous networks, either heavy burdens on base stations or serious energy waste. In order to ensure coverage of the network while reducing the total energy consumption, we adopt a green mobile cyberphysical system (MCPS) to handle this problem. In this paper, we propose a feature extraction method using sliding window to extract the distribution feature of mobile user equipment (UE), and a case study is presented to demonstrate that the method is efficacious in reserving the clustering distribution feature. Furthermore, we present traffic clustering analysis to categorize collected traffic distribution samples into a limited set of traffic patterns, where the patterns and corresponding optimized control strategies are used to similar traffic distributions for the rapid control of base station state. Experimental results show that the sliding window is more superior in enabling higher UE coverage over the grid method. Besides, the optimized control strategy obtained from the traffic pattern is capable of achieving a high coverage that can well serve over $98 \%$ of all mobile UE for similar traffic distributions.
\end{abstract}

\section{Introduction}

The rising demands for network resources and quality of service are forcing operators of wireless cellular networks to continuously add capacities to their networks. One of the means to do so is densification: deploying heterogeneous networks with a multitude of smaller base stations, such as picobase stations and femtobase stations [1-4]. Such a heterogeneous network will be significantly more complex than today's system and hence will require more effective state control strategies to achieve energy efficient coverage. The control strategy decides the active or sleep state of each base station in the network. If all base stations were active when the traffic demand is low, the unnecessary energy consumption can be significant $[5,6]$. On the other hand, excessively frequent switching on/off the base stations is not practical, considering the control precision, protocol limitation, and lifespan of the stations. Therefore, the control strategy of a heterogeneous network must be optimized to reduce energy consumption, while maintaining its QoS including coverage, response time, and spectral efficiency.

To determine an optimized state control strategy for a network, it is necessary to capture its traffic distribution, that is, the geographical distribution of mobile UE in the network [7]. Uniform Poisson modeling method was presented based on IMT-advanced evaluation guidelines in [8]. Although very popular, this method is less effective when representing the heterogeneous user activities and geographical characteristics of the network using only Poisson point processes. References $[9,10]$ modeled traffic distribution using a two-dimensional sub-Poisson point process in order to capture the heterogeneity; that is, a perfect lattice and a 


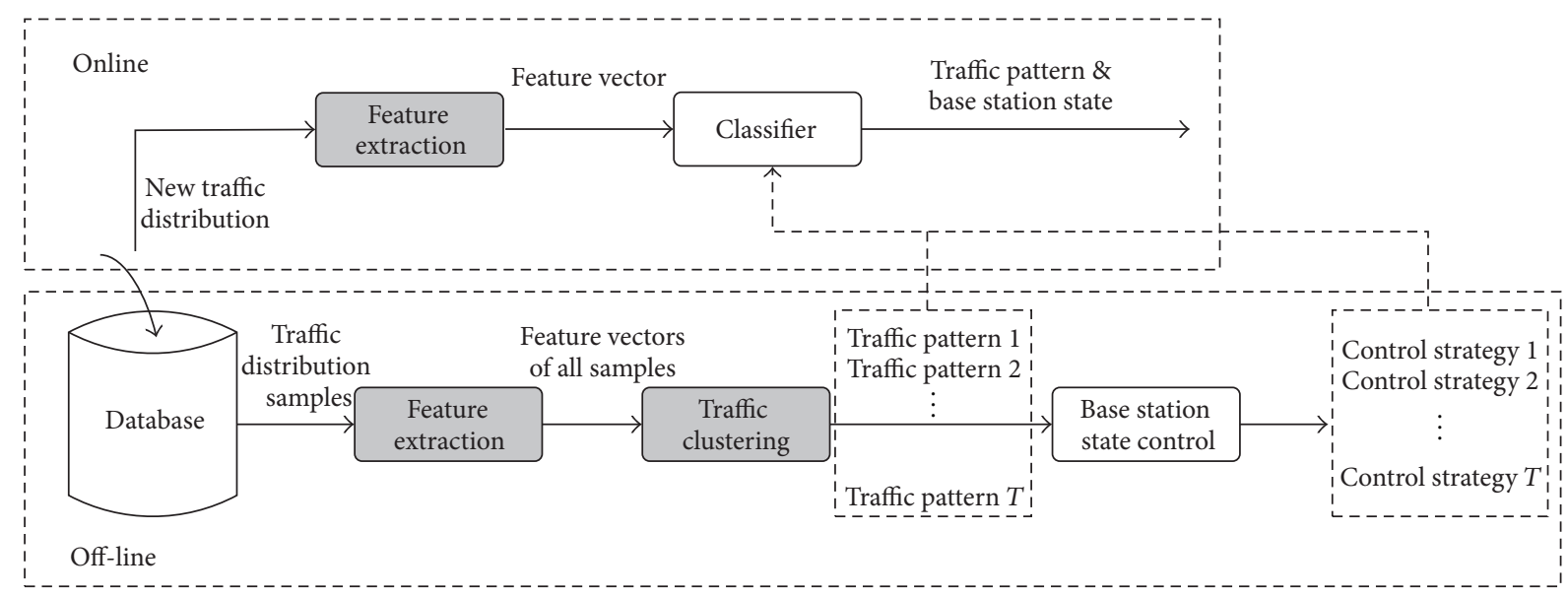

FIGURE 1: A green mobile cyberphysical system framework.

random perturbation were applied to generate sub-Poisson UE distribution. A thorough review of point processes was presented in [11], in which clustering properties of point processes in space are compared. In [12], Mirahsan et al. introduced a heterogeneous traffic modeling method that allows statistical adjustment. This method is continuously scalable from uniform to heterogeneous point process.

Optimization algorithms were developed to determine control strategies while satisfying a set of conflicting requirements such as coverage and energy efficiency. In [13], a nonlinear integer programming method was used to determine optimized control strategy. In [14], Lorincz et al. used an efficient linear integer programming method. In [15], Jung et al. presented a two-step optimization algorithm that identified the minimal set of base stations needed to maintain coverage and analyzed the bandwidth and power allocation of each base station to minimize energy consumption. Minimax algorithm was adopted in the development of a distributed base stations switch-off method in [16], while the optimization problem was solved using time-consuming exhaustive search method. In [17], Al-Kanj et al. proposed a green radio network planning approach which jointly optimized the number of active base stations and the base station on/off switching strategies. It is worth noting that most of the optimization approaches considered only the traffic distribution at a certain time. Monitoring the dynamic traffic distributions and controlling base stations in a real-time manner are not yet achieved. Mobile cyberphysical systems (MCPSs) were presented for this purpose.

A MCPS is a combination of computation and communication systems. It is capable of sensing, processing, and responding to the dynamic changes of traffic distribution of a network [18-21]. MCPS inherits many essential and important characteristics of traditional cyberphysical system (CPS) [22], such as intelligent network, interaction between human and MCPS, and computation with physical process [23]. It is also capable of integrating wireless sensor network (WSN) and cloud environment. For the tasks requiring more resources than what is available locally, MCPS gives customers rapid access to other WSN and clouds [24]. In a
MCPS, mobile UE, such as smart phones and tablets, acts like cyber terminals with storages and processing capabilities. Sensors in the system like GPS and Bluetooth collect physical data such as the geographical positions of UE in the system and their receiving power from the surrounding base stations $[25,26]$. The data is transmitted to the computational backbone of MCPS, the clouds. The clouds analyze the data to determine optimal control strategies for base stations, resource allocation, and network scheduling.

In [27], we presented a green MCPS. To maximize the energy efficiency of the network, a heuristic method was developed to determine the control strategy according to the dynamic traffic distributions. The two major components of the green MCPS are introduced in this paper: (1) Modeling: we use a sliding window approach to extracting the overlapped features from traffic distributions; (2) Optimization: a traffic clustering algorithm is presented to classify all the samples of traffic distributions into a set of traffic patterns. The patterns and corresponding optimized control strategies will be used to handle new traffic distributions collected in real time.

The remainder of the paper is organized as follows. The framework of the green MCPS is explained in Section 2. The feature extraction and traffic clustering algorithms are introduced in detail in Section 3. A set of experiments are presented to validate the algorithms in Section 4. Section 5 concludes the paper.

\section{Green MCPS Framework}

The framework of the green MCPS is shown in Figure 1. It consists of two processes, online and off-line.

The off-line process is a learning process. Its input is a set of traffic distributions sampled at different times when the network is deployed. The outputs of this process are a set of traffic patterns and the corresponding optimized control strategies of the base stations. When the off-line process starts, a set of traffic distribution samples have been collected at various times and saved in the database of the system. Each sample contains the geographical locations of all mobile UE 


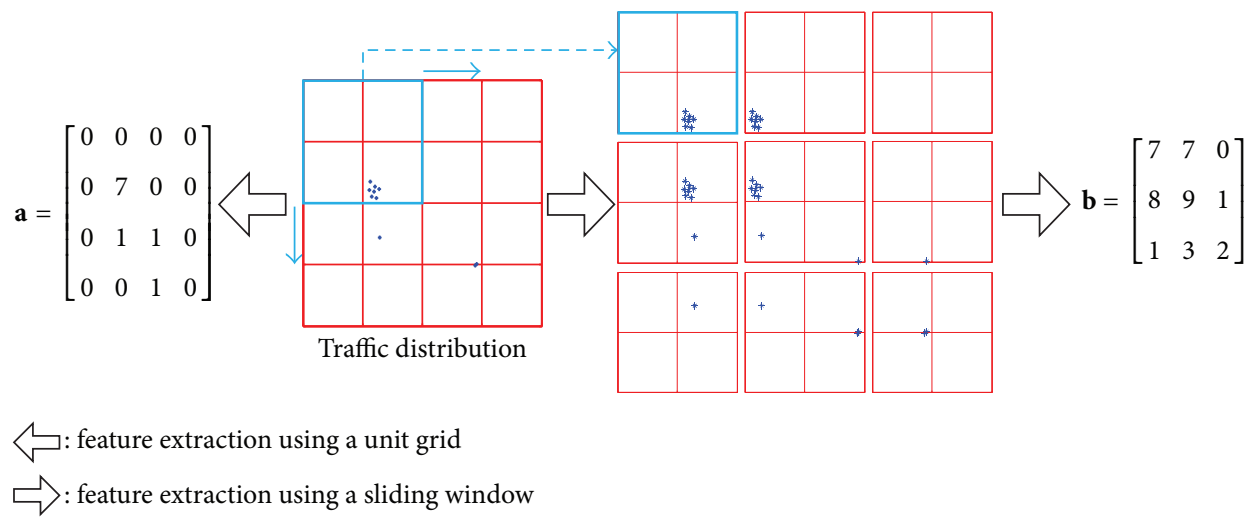

FIGURE 2: Feature extraction example.

in the 2-dimensional target region at certain time. It must be noted that we must collect enough samples in order to capture the characteristics of the network's traffic distribution during its daily operation. These samples are then transferred to the feature extraction module, in which if the target region is partitioned into equal-sized areas, a feature vector with a dimension equal to the number of areas is used to represent the UE densities in each area. The detailed feature extraction algorithm will be explained in the next section. After that, the feature vectors are transferred to a clustering module in order to group all traffic distribution samples into a certain number of traffic patterns using the clustering algorithm, as introduced in the following section. Each pattern is a traffic distribution that represents a group of samples with similar characteristics of geographical distribution. Each pattern has an optimal control strategy for the target region. The optimized strategy is decided using a heuristic method as introduced in previous papers [27]. The traffic patterns and the corresponding control strategies are stored to the database.

The online process starts when a new traffic distribution is identified. In this case, the system starts the feature extraction module to extract and represent its distribution feature into a feature vector. Then the vector is imported into a classifier, which acts as a decision-making tool that is capable of matching the sample with the traffic patterns according to the Euclidean distance between feature vectors [28]. If the Euclidean distance of the new feature vector is with a defined range from that of a pattern, the sample is matched to the pattern and the corresponding control strategy is used to control the base stations of the new sample. Therefore, the control strategies of the new distribution can be rapidly obtained. If there is no match, the new sample will be stored into the database waiting for the next matching.

\section{Feature Extraction and Traffic Clustering Algorithms}

3.1. Feature Extraction Using Sliding Window. If we partition a 2-dimensional target region into equal-sized grids, that is, unit grid, and count the amount of mobile UE within each grid, we can represent the characteristics of the traffic distribution into a feature vector. However, elements of the feature vector are always independent of each other. Since the detail of the feature vector is limited by the grid size, the unit grid method is not effective in capturing the clustering characteristics of the traffic distribution.

The high density distribution of mobile UE forms cluster, a common and critical distribution form to affect the coverage and QoS of heterogeneous networks [7]. In order to effectively capture the clustering characteristics and detect changes in traffic distribution, we propose a new method to represent traffic distribution. We use a sliding window with a sliding step smaller than the length of the window instead of using even grids. Using a traffic distribution as shown in Figure 2 as an example, the feature matrix captured using $4 \times 4$ red grids is a. A square sliding window is defined as being as large as $2 \times 2$ grids, shown as the blue cell in Figure 2, and a sliding step size is the same as the grid size. The sliding window slides from left to right until it reaches the right end of the region. Then the window slides down one step and scans from left to right. The process stops when the whole target region is scanned, and the resulting matrix is b. It can be seen that a cluster is formed in the target region. This clustering feature is represented by element 7 of matrix a, while four elements (7, 7, 8, and 9) of matrix $\mathrm{b}$ reserve the paradigm of this cluster similarly. If there are clusters of UE distributions, the sliding window method creates the feature matrix with more elements to depict the clustering distribution, which has the advantage of reserving the integrity of clustering distribution in case of the loss of a cluster in the process of feature extraction. In the sliding window method, both the window size and sliding step size are important design parameters.

Given a sample of traffic distribution in a region with area $L^{2}$, it contains $N_{u}$ mobile UE and the coordinate of mobile UE $k$ is $\left(x_{k}, y_{k}\right), k \leq N_{u}$. In order to extract the clustering characteristics, we partition the target region into $N_{g} \times N_{g}$ equal-sized grids, and the size of grid is $e=L / N_{g}$. The size of the sliding window $e_{w}$ and sliding step size $s_{w}$ are given as the integer multiple of $e$ for the convenience of data processing, denoted as $e_{w}=p e\left(p \in \mathbf{z}^{+}, \mathbf{z}^{+}\right.$is a set of positive integers) and $s_{w}=q e\left(e_{w}=p e, p \in \mathbf{z}^{+}\right)$. Besides, the sliding step size should be smaller than the window size; both of them should 


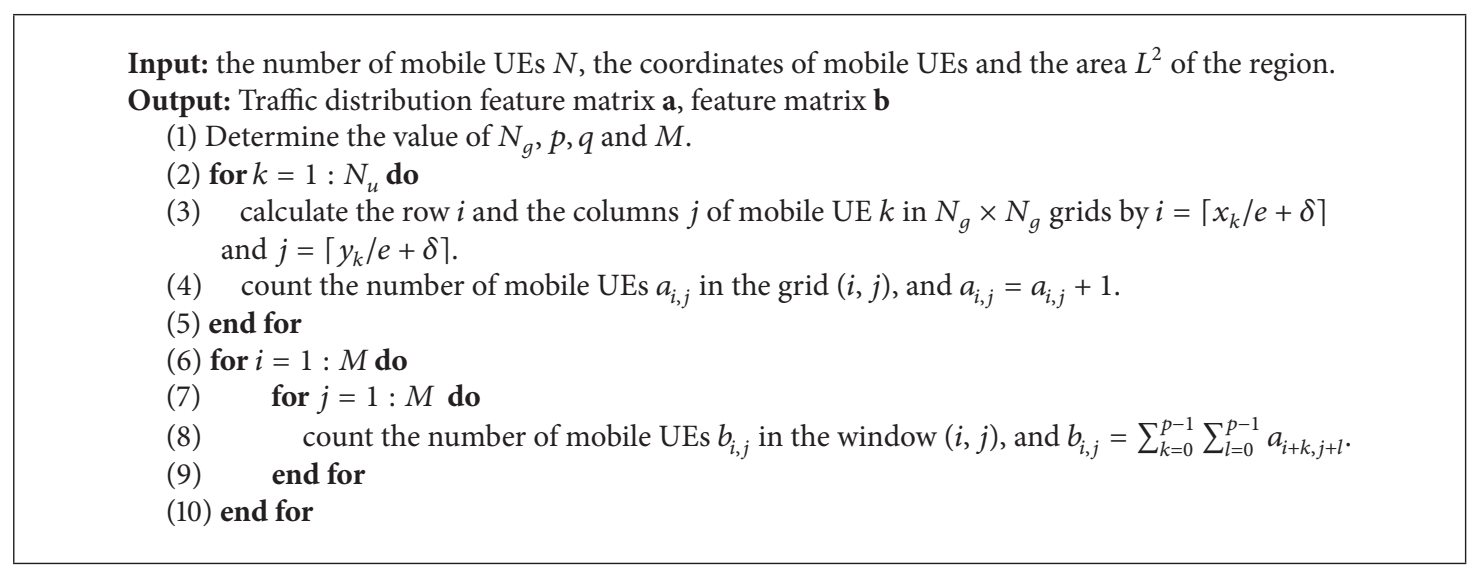

Algorithm 1: The feature extraction using sliding window.

be smaller than the target region. That is, $0<q<p<N_{g}$. If a window needs to slide $M$ times to cover the length of the region $L$, we have

$$
L=N_{g} e=M q e+p e, \quad\left(0<q<p<N_{g}\right) .
$$

In general, $q$ is a small integer. Given $q, M$ and $p$ are

$$
\begin{aligned}
M & =\left[\frac{N_{g}}{q}\right]-n_{0} \\
p & =N_{g}-M q,
\end{aligned}
$$

where a small integer $n_{0} \in \mathbf{z}^{+}$is added to ensure that $q<$ $p$, and it is usually assigned manually. In fact, the optimal value of $p$ and $q$ can only be determined after numerical experiments. With the above variables, the feature extraction method is presented as shown in Algorithm 1 .

In Algorithm $1, \delta$ is defined to keep both $i$ and $j$ from becoming 0 , and its value is infinitesimal. Matrix $\mathbf{a} \in \mathbf{R}^{N_{g} \times N_{g}}$ is the traffic distribution feature matrix obtained using $N_{g} \times$ $N_{g}$ grids. Matrix $\mathbf{b}$ is obtained using the sliding window method.

Subsequently, we convert matrix b to feature vector $\mathbf{s}$ with $M^{2}$ elements by array rearrangement; this function is implemented using the reshape function of MATLAB. In the off-line process, after conducting feature extraction for $N$ traffic distribution samples, we obtained feature vector set $\mathbf{s}=\left\{s_{1}, s_{2}, \ldots, s_{N}\right\}$, which will be transferred to the following clustering module. In order to process data conveniently, we normalize these vectors.

3.2. Traffic Clustering. Traffic clustering module categorizes all traffic distributions samples into a limited set of traffic patterns. Clustering is an unsupervised classification approach, which is capable of analyzing the internal characteristic and mutual relationship of objects without label $[29,30]$. The $K$ means clustering is a well-known algorithm for classification based on the distance measurement and the squared error [31, 32]. Nevertheless, it has significant shortcomings, including predetermined number of clusters, unguaranteed global optimum, and being sensitive to noises. $K$-medoids algorithm [33] uses the medians of a cluster as its centroid to reduce the influence of noises. Kernel $K$-means algorithm is presented in [34], which transforms original features set of objects into a higher-dimensional space to make objects more separable. Spectral clustering method performs dimensionality reduction using Laplacian eigenmap [35]. Other advanced clustering algorithms include iterative self-organizing data analysis technique (ISODATA), Gaussian mixture (GM), and density based spatial clustering of applications with noise (DBSCAN). Considering the computation complexity and multidimensional features of the traffic distributions, we decide to combine the $K$-means and the spectral clustering algorithm presented in [36] to analyze the internal similarity of traffic distribution vectors. The process of traffic clustering primarily comprises two parts, the determination of optimal number of traffic patterns and the classification of all traffic distribution vectors, as presented in Algorithm 2.

In Algorithm 2, the optimal number of traffic patterns $K(1<K<N)$ is decided using the average silhouette method. The silhouette value measures how well a sample lies within its cluster comparing to other clusters [37]. In order to obtain the silhouette value of each sample, we classify all samples into $k$ clusters at first, where $k$ is a variable quantity. For each sample $i$, let $a(i)$ be the average dissimilarity of $i$ to all other samples within the same cluster, and the average dissimilarity of sample $i$ can be defined as the average of the distance from $i$ to all other samples within the same cluster. $b(i)$ denotes the lowest average dissimilarity of $i$ to any other sample in other clusters. Then, the silhouette value can be obtained by combining $a(i)$ and $b(i)$.

$$
s(i)=\frac{b(i)-a(i)}{\max \{a(i), b(i)\}} .
$$

From the above definition, it can be seen that $-1<s(i)<1$ for each sample $i$.

$s(i)$ is close to 1 for $a(i) \ll b(i)$, which indicates that sample $i$ is well clustered because $a(i)$ represents how dissimilar $i$ is to its own cluster. When $s(i)$ is around zero for $a(i)=b(i)$, sample $i$ is on the border of two clusters. The worst situation takes place when $s(i)$ is close to -1 when $a(i) \gg b(i)$, 
Input: Feature vectors of all traffic distribution samples $\mathbf{s}=\left\{s_{1}, s_{2}, \ldots, s_{N}\right\}$.

Output: Traffic patterns $T_{1}, T_{2}, \ldots, T_{K}$

(1) Normalize Feature vectors to $\mathbf{s}^{\prime}=\left\{s_{1}^{\prime}, s_{2}^{\prime}, \ldots, s_{N}^{\prime}\right\}$.

(2) Determine the number of traffic patterns $K$ by the average silhouette method.

(3) Construct an affinity matrix A with Gaussian kernel function, in which $A_{i j}=\exp \left(-d^{2}\left(s_{i}^{\prime}, s_{j}^{\prime}\right)\right) / 2 \delta^{2}$ holds for $i \neq j$ and $A_{i i}=0$.

(4) Define the diagonal degree matrix $\mathbf{D}\left(D_{i i}=\sum_{j} A_{i j}\right)$. Normalize the affinity $\mathbf{A}$ to $\mathbf{L}$, and $\mathbf{L}=\mathbf{A}^{-1 / 2} \mathbf{D} \mathbf{A}^{-1 / 2}$.

(5) Compute the first $K$ eigenvectors of $\mathbf{L}$. Construct a matrix $\mathbf{X}=\left\{x_{1}, x_{2}, \ldots, x_{K}\right\} \in \mathbf{R}^{N \times K}$.

(6) Construct a matrix $\mathbf{Y}$ from $\mathbf{X}$ by normalizing the rows of $\mathbf{X}$ to norm 1 , and $Y_{i j}=X_{i j} /\left(\sum_{j} X_{i j}^{2}\right)^{1 / 2}$.

(7) Treating each row of $\mathbf{Y}$ as a point, cluster them into $K$ traffic patterns by $K$-means.

(8) Assign the original feature vector $s_{i}$ of traffic distribution sample to traffic pattern $j$ according to the assigned label $j$ of the row $i$ of the matrix $\mathbf{Y}$.

(9) Compute the features of traffic patterns, and $u_{j}=1 /\left|T_{j}\right| \sum_{s_{i} \in T_{j}} s_{i}(j=1,2, \ldots, K)$.

Algorithm 2: The traffic clustering algorithm.

meaning that the sample is misclassified. Furthermore, the average silhouette $s_{\text {ave }}$ is the average of silhouette $s(i)$ of all samples for a traffic pattern; it shows how tightly all samples are grouped in the cluster and, hence, evaluates clustering validity. If there are too many or too less clusters, some narrow silhouette may occur; thus it is used to determine the optimal number of clusters. It is believed that an optimal number of traffic patterns $K$ is the one at which the average silhouette is maximized over a range of values for $k$ [38].

In order to obtain the optimal $K$ traffic patterns, the spectral clustering method [36] is applied. First, an affinity matrix A is formed, where $d\left(s_{i}^{\prime}, s_{j}^{\prime}\right)$ is the Euclidean distance between $s_{i}^{\prime}$ and $s_{j}^{\prime}$, and $\delta^{2}$ is the scaling parameter that determines the speed of the affinity matrix falling off. Then, the selection of $K$ eigenvectors of $\mathbf{L}$ leads to the feature dimensionality reduction. Finally, $K$-means algorithm is adopted to assort all traffic distribution samples into various traffic patterns.

After traffic clustering, the optimal control strategy for each pattern is decided using the method presented in our previous work [27]. Briefly, considering the constraint conditions of the $\mathrm{UE}$ association, the received signal to interference and noise ratio (SINR) of UE, and capacity of base station, the optimal control strategy maximizes the energy efficiency of a traffic pattern. Assume that $N_{u} \mathrm{UE}$ is scattered in the region under traffic pattern $t$ and $N_{b}$ base stations are deployed as well. The UE associate is denoted as $s_{i, k}^{t}$, in which $s_{i, k}^{t}$ represents that UE $k$ is $\left(s_{i, k}^{t}=1\right)$ or is not $\left(s_{i, k}^{t}=0\right)$ associated with base station $i$ under traffic pattern $t$. The SINR of UE $k$ from base station $i$ is denoted as $\gamma_{i, k}^{t}$. $M_{i}^{\max }$ represents the maximum amount of servable UE for base stations $i$. For each traffic pattern, the control strategy is represented using vector $\mathbf{a}^{t}=\left[a_{i}^{t}\right]_{1 \times N_{b}}$, where $a_{i}^{t}$ denotes that the state of base station $i$ is active $\left(a_{i}^{t}=1\right)$ or sleep $\left(a_{i}^{t}=0\right)$. Hence, the optimal control model can be formulated as follows:

$$
\begin{aligned}
& \max \eta_{E E} \\
& =\frac{\sum_{i=1}^{N_{b}} a_{i}^{t} \sum_{k=1}^{N_{u}} s_{i, k}^{t} R_{i, k}^{t}}{\sum_{i=1}^{N_{b}}\left[a_{i}^{t}\left(k_{i} P_{i}^{\max } \sum_{k=1}^{N_{u}} s_{i, k}^{t} / M_{i}^{\max }+P_{i}^{A}\right)+\left(1-a_{i}^{t}\right) P_{i}^{S}\right]},
\end{aligned}
$$

where $R_{i, k}^{t}$ is the transmission rate calculated using $R_{i, k}^{t}=$ $\log _{2}\left(1+\gamma_{i, k}^{t}\right)$. $k_{i}$ denotes the power amplifier inefficiency factor. $P_{i}^{\max }$ represents the total transmit power. $P_{i}^{A}$ is the circuit power consumption of active base station $i$ and $P_{i}^{S}$ is the circuit power consumption when it is in sleep mode. A heuristic algorithm is adopted to solve the problem, and the states of all base stations, the number of active base stations, and the association of UE with base stations can be obtained eventually [27].

\section{Simulation Results and Discussions}

The presented method is demonstrated using a $1600 \mathrm{~m} \times$ $1600 \mathrm{~m}$ target region. In order to verify the efficacy of the presented method in different traffic distributions, we create 1000 different traffic distributions including three distribution models:

(i) A rand function in MATLAB is used to generate a complete random distribution (Poisson).

(ii) A perfect lattice and a random perturbation [10] are used to generate a uniform distribution (subPossion).

(iii) Thomas process [11] is used to generate a clustered distribution (sub-Possion).

Each of these traffic distribution samples consists of a random amount of UE between 250 and 650. In order to differentiate the samples, the difference of the UE numbers between any two samples is at least 50.113 base stations are in cochannel deployment in a two-tier heterogeneous cellular network, including one macro base station and 112 small base stations. In addition, other major network parameters, such as the bandwidth and transmission power of base stations, bit error rate, and outage probability, are set referring to [27].

According to the optimal control strategy, the amount of mobile UE served by the active base stations in each sample is counted. Hence, we select the rate of UE coverage as the 


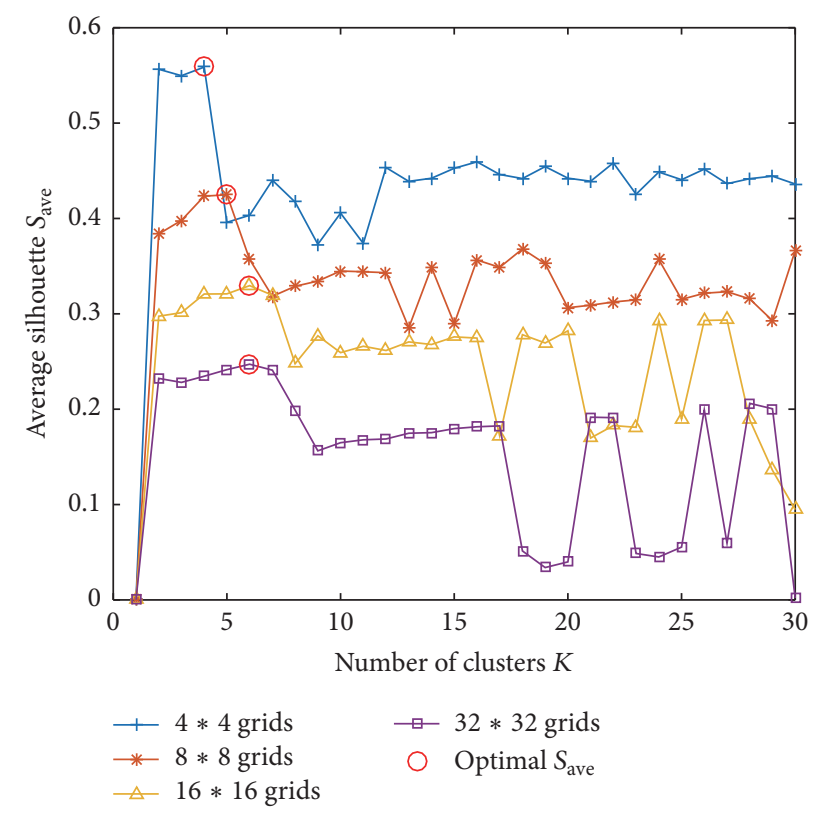

Figure 3: Average silhouettes value for different number of traffic patterns.

performance metric of green MCPS.

$$
\eta=\frac{n_{s}}{N_{u}},
$$

where $n_{s}$ is the amount of mobile UE served by the active base stations and $N_{u}$ is the total mobile UE in the target region. According to [27], the UE coverage for a traffic pattern should be more than $98 \%$ in order to satisfy the required QoS. In this paper, we believe $98 \%$ or higher coverage is a "good coverage."

We start by estimating an optimal number of traffic patterns. The sliding window size $e_{w}$ is predefined as $2 e$ ( $e$ is the size of a grid) and the sliding step size is $e$. The average silhouettes $s_{\text {ave }}$ of 1000 traffic distribution samples for different number of traffic patterns and different partition grids are illustrated in Figure 3.

It is observed that the average silhouettes value achieves their peak over a range of possible value for $k$ from 1 to 30. Thus, the optimal number of traffic patterns can be determined as the corresponding value of $k$ at the peak. That is, the optimal number of traffic patterns is 4 when the partitioned grids are $4 \times 4,5$ when the grids are $8 \times 8$, and 6 when the grids are $16 \times 16$ and $32 \times 32$. It is noted that the number of traffic patterns at the optimal average silhouettes has slightly been raised but not sharply changed with the increasing of the number of traffic patterns. Hence, an appropriate number of traffic patterns clustered can be determined to be 6 . The performance analysis is carried out for different number of traffic patterns next.

4.1. Feature Extraction Using Sliding Window with Different Sliding Step Sizes. In this experiment, we count the rate of traffic distribution samples with UE coverage over $98 \%$ based on the feature vectors extracted by sliding window with different step sizes and a unit grid. The number of grids

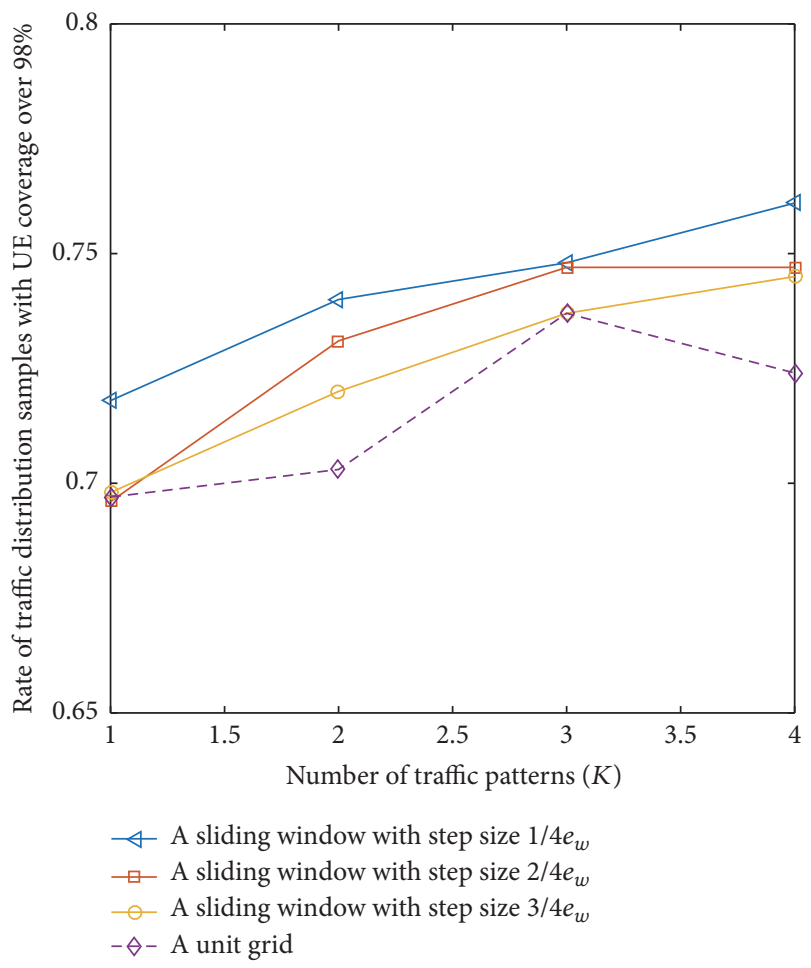

FIGURE 4: Feature extraction using sliding window with different step sizes and a unit grid.

partitioned is set to be $16 \times 16$, and the size of the sliding window $e_{w}$ is four times the size of a grid $\left(e_{w}=4 e\right)$. Thus, the value of $q$ can be 1,2 , or 3 .

We can observe from Figure 4 that the smaller the sliding step size is, the more the traffic distribution samples achieve coverage over $98 \%$. The percentage of samples with good coverage (at least $98 \%$ UE coverage) at step size $1 / 4 e_{w}$ is always higher than others. That is, sliding window with smaller sliding step size, we can not only extract more features but also reserve the clustering feature more faithfully. This leads to a good coverage. In addition, it shows that, by using the proposed sliding window method, we can always acquire better coverage than that of using the unit grid, shown with the dotted line on the figure.

4.2. UE Coverage under Different Sliding Window Sizes and Traffic Patterns. In order to discuss the effects of sliding window size and the number of traffic patterns on the coverage, we use $32 \times 32$ grids to partition the region, and grid edge is $e$. The sliding step size $s_{w}$ is always half of the window size $e_{w}$. Three different intervals of UE coverage rate are counted in Figure 5, namely, [0, 0.9], [0.9, 0.98], and $[0.98,1]$, respectively.

It can be seen that the smaller the size of the sliding window is, the higher the percentage of samples achieves good coverage. This is because that more heterogeneous distribution features are preserved by using a smaller sliding window, which contributes to better coverage. However, smaller window size means larger feature vector for a sample, and this unavoidably reduces the operational speed. It is 

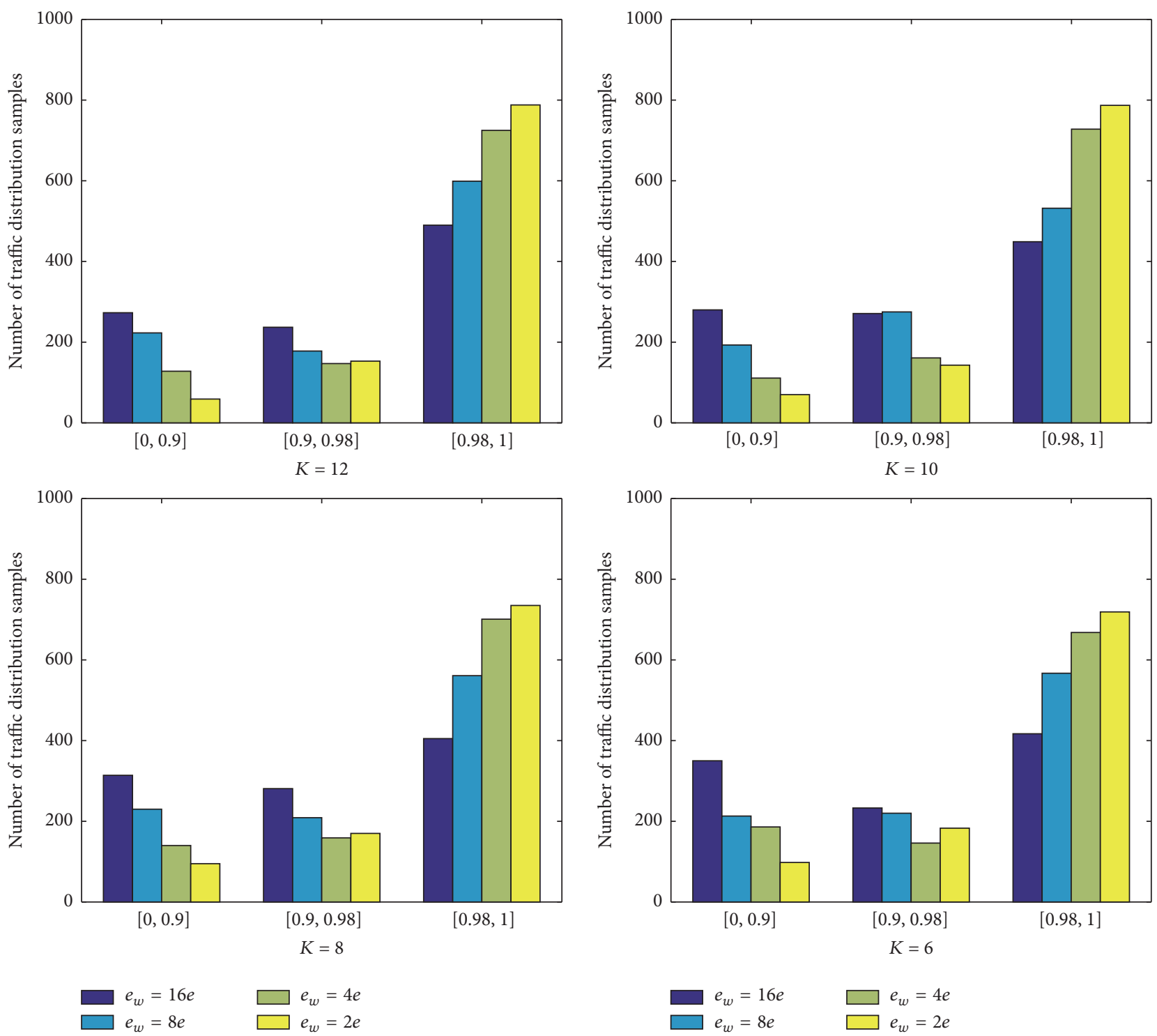

Figure 5: Coverage for different number of patterns.

necessary to compromise between the coverage and operational speed when we decide the window size in practice. We can choose different window size according to different requirements for the rates of coverage. At the same time, we can observe that, by increasing the number of traffic patterns, the number of samples with good coverage rises slightly but is not significant. Therefore, we conclude that the number of traffic patterns is less important than other factors such as sliding step size and window size. In practice, we can decide the pattern number by the average silhouette method.

\subsection{Number of Active Base Stations for Different Traffic} Clusters. After adopting the optimal control strategy of the traffic pattern to its samples, we can observe the number of active base stations in the traffic distribution samples. Here, in the process of feature extraction, $32 \times 32$ grids are used, the size of the sliding window $e_{w}$ is $2 e$, and the sliding step size is $e$.
As shown in Figure 6, the difference between the maximal and minimal number of active base stations for all traffic distribution samples within the same traffic pattern is shown. That is, most of the number of active base stations for a traffic pattern is slightly more than that of the maximal number of active base stations for samples in this pattern. This means that applying the control strategy of a traffic pattern to the samples belonging to this pattern will ensure good coverage, and this can achieve significant energy saving as well.

4.4. SINR Distribution under a Traffic Pattern. Finally, a SINR distribution example under a traffic pattern is depicted in Figure 7, in order to observe the coverage of active base stations.

In this traffic pattern, UE is represented as dots, and base stations are uniformly distributed within the target region. As shown in Figure 7, the triangles represent small base 

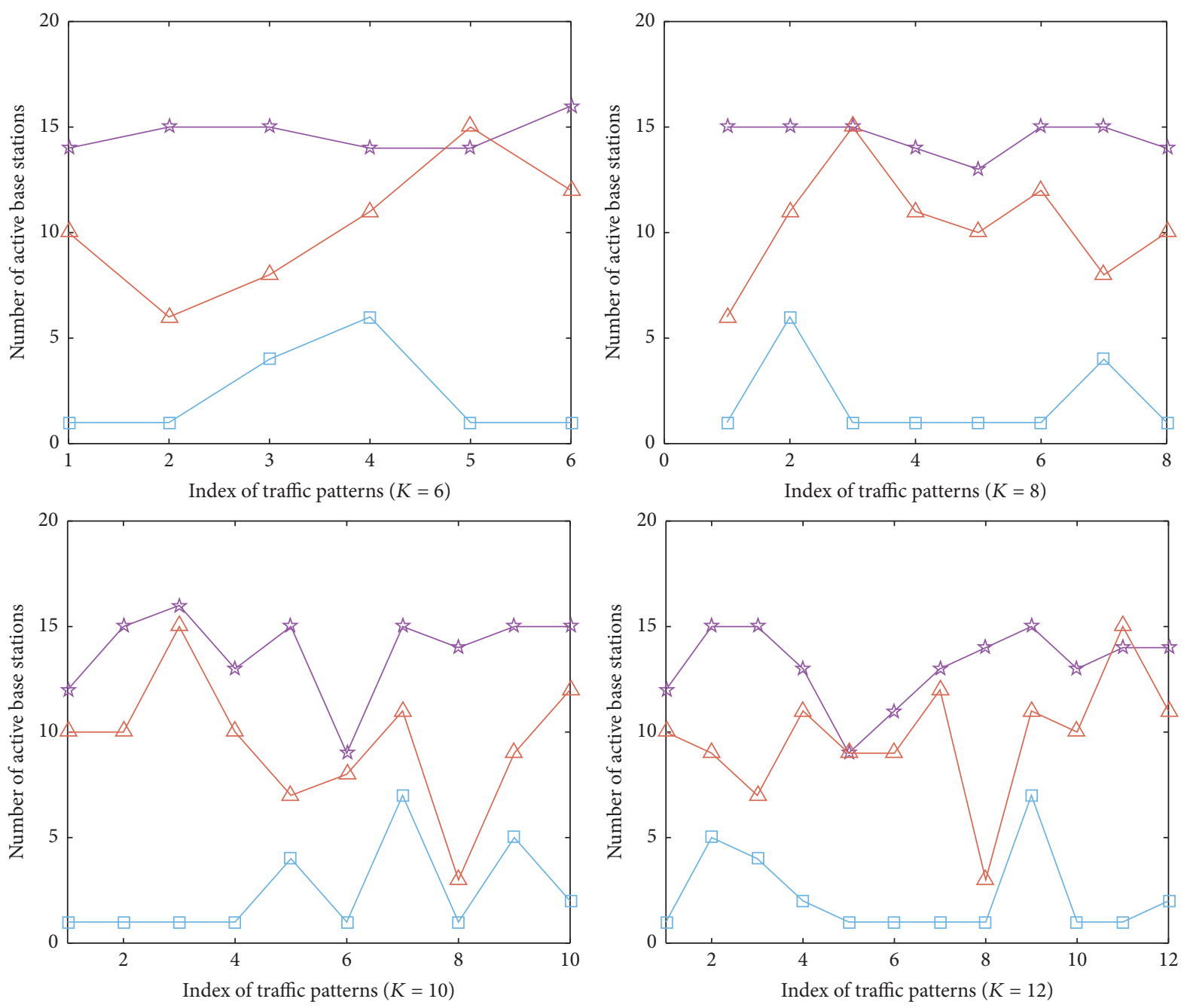

Number of active base stations for traffic patterns

$\triangle$ Maximal number of active base stations for samples

$\square$ Minimal number of active base stations for samples

Number of active base stations for traffic pattern

$\triangle$ Maximal number of active base stations for samples $\square$ Minimal number of active base stations for samples

Figure 6: Number of active base stations for different traffic clusters.

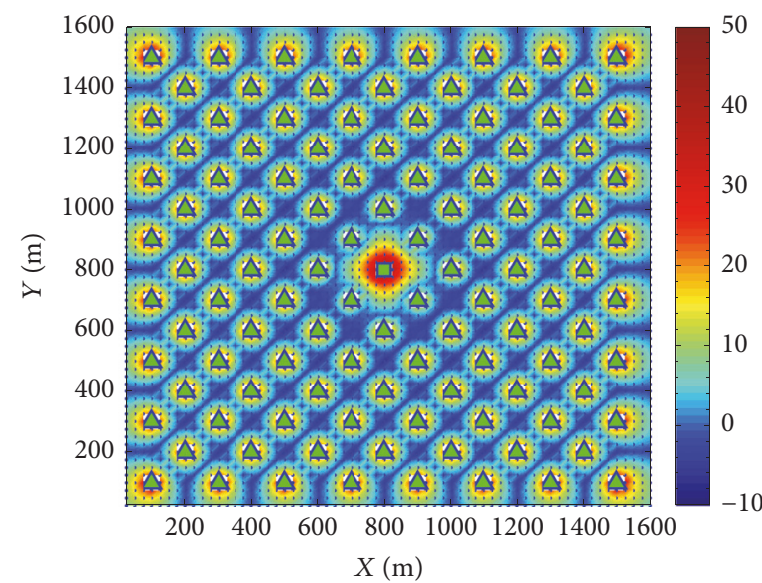

(a) Initial state

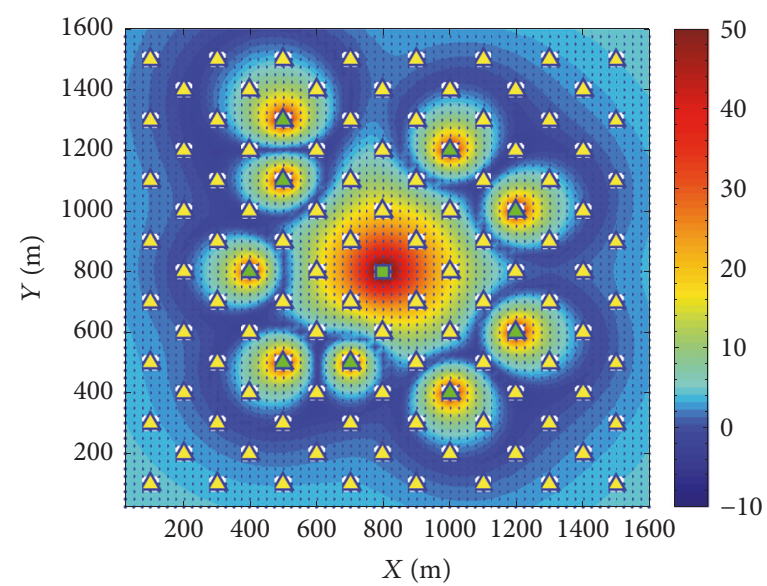

(b) Final state

FIGURE 7: SINR distributions. 
stations, and the squares represent macro base station. The state of base stations is represented by colors, where yellow means sleep and green means active. The values of SINR are in the range of $-10 \mathrm{~dB}$ to $50 \mathrm{~dB}$, which are represented by different gradient colors. Figure 7 (a) shows the initial SINR distribution of UE when all base stations are active. We can observe that the coverage of active base stations is limited due to the ubiquitous interference between base stations. After adopting the optimal control strategy, only ten base stations are activated, and the coverage with higher SINR of UE around the active base stations increases in Figure 7(b). This indicates that the coverage of active base station is enlarged under the condition of over $98 \%$ UE coverage.

\section{Conclusion}

In this paper, we present a feature extraction method using sliding window for traffic distributions in a green mobile cyberphysical system. The method has the advantages of reserving more clustering distribution features over using the grid method. In order to implement rapid base station state control and extend the lifespan of a heterogeneous network, we apply clustering analysis for all traffic distributions to obtain a limited set of traffic patterns. Numerical results demonstrate that the proposed method helps obtain better UE coverage comparing with using the grid method. It is worth noting that both smaller sliding step size and smaller sliding window size can lead to good UE coverage but slow the operational speed of the network.

\section{Conflicts of Interest}

The authors declare that they have no conflicts of interest.

\section{Acknowledgments}

This research was supported in part by the National Natural Science Foundation of China (Grant no. 61601482) and Guangdong Technology Project (2016B010125003 and 2016B010108010) and sponsored by the Foundation of Science and Technology on Information Transmission and Dissemination in Comm. Networks Lab, National Key Laboratory of Anti-Jamming Communication Technology, and State Joint Engineering Laboratory for Robotics and Intelligent Manufacturing funded by National Development and Reform Commission (no. 2015581).

\section{References}

[1] J. F. Monserrat, I. Alepuz, J. Cabrejas et al., "Towards usercentric operation in 5G networks," Eurasip Journal on Wireless Communications and Networking, vol. 2016, no. 1, article no. 6, pp. 1-7, 2016.

[2] J. F. Monserrat, G. Mange, V. Braun, H. Tullberg, G. Zimmermann, and Ö. Bulakci, "METIS research advances towards the 5G mobile and wireless system definition," Eurasip Journal on Wireless Communications and Networking, vol. 2015, no. 1, pp. 1-16, 2015.
[3] L. Zhou, Z. Sheng, L. Wei et al., "Green cell planning and deployment for small cell networks in smart cities," Ad Hoc Networks, vol. 43, pp. 30-42, 2016.

[4] L. Zhou, C. Zhu, R. Ruby et al., "QoS-aware energy-efficient resource allocation in OFDM-based heterogenous cellular networks," International Journal of Communication Systems, vol. 30, no. 2, p. e2931, 2017.

[5] M. Hoshino, Y. Yuda, T. Takata, and A. Nishio, "Performance evaluation of jt-comp under non full buffer traffic condition on heterogeneous network with dense small cells," vol. 112, pp. 2934, 2012.

[6] L. Zhou, X. Hu, E. C.-H. Ngai et al., "A dynamic graph-based scheduling and interference coordination approach in heterogeneous cellular networks," IEEE Transactions on Vehicular Technology, vol. 65, no. 5, pp. 3735-3748, 2016.

[7] M. Mirahsan, Z. Wang, R. Schoenen, H. Yanikomeroglu, and M. St-Hilaire, "Unified and non-parameterized statistical modeling of temporal and spatial traffic heterogeneity in wireless cellular networks," in Proceedings of 2014 IEEE International Conference on Communications Workshops, ICC 2014, pp. 55-60, aus, June 2014.

[8] ITU-R, "Guidelines for evaluation of radio interface technologies for imt-advanced," M.2135-1, 2009.

[9] J. Rataj, I. Saxl, and K. Pelikán, "Convergence of randomly oscillating point patterns to the Poisson point process," Applications of Mathematics, vol. 38, no. 3, pp. 221-235, 1993.

[10] V. Lucarini, "From symmetry breaking to Poisson point process in 2D Voronoi tessellations: the generic nature of hexagons," Journal of Statistical Physics, vol. 130, no. 6, pp. 1047-1062, 2008.

[11] B. Błaszczyszyn and D. Yogeshwaran, "Clustering comparison of point processes, with applications to random geometric models," Lecture Notes in Mathematics, vol. 2120, pp. 31-71, 2015.

[12] M. Mirahsan, R. Schoenen, and H. Yanikomeroglu, "HetHetNets: Heterogeneous Traffic Distribution in Heterogeneous Wireless Cellular Networks," IEEE Journal on Selected Areas in Communications, vol. 33, no. 10, pp. 2252-2265, 2015.

[13] K. Son, S. Nagaraj, M. Sarkar, and S. Dey, "QoS-aware dynamic cell reconfiguration for energy conservation in cellular networks," in Proceedings of 2013 IEEE Wireless Communications and Networking Conference, WCNC 2013, pp. 2022-2027, chn, April 2013.

[14] J. Lorincz, A. Capone, and D. Begušić, "Optimized network management for energy savings of wireless access networks," Computer Networks, vol. 55, no. 3, pp. 514-540, 2011.

[15] H.-S. Jung, H.-T. Roh, and J.-W. Lee, "Energy and traffic aware dynamic topology management for wireless cellular networks," in Proceedings of 2012 IEEE International Conference on Communication Systems, ICCS 2012, pp. 205-209, sgp, November 2012.

[16] X. Su, E. Sun, M. Li, F. R. Yu, and Y. Zhang, "An Energy-Efficient User Location-Aware Switch-Off Method for LTE-A Cellular Networks," Wireless Personal Communications, vol. 84, no. 3, pp. 1817-1833, 2015.

[17] L. Al-Kanj, W. El-Beaino, A. M. El-Hajj, and Z. Dawy, "Optimized joint cell planning and BS on/off switching for LTE networks," Wireless Communications and Mobile Computing, vol. 16, no. 12, pp. 1537-1555, 2015.

[18] X. Hu, T. H. S. Chu, H. C. B. Chan, and V. C. M. Leung, "Vita: a crowdsensing-oriented mobile cyber-physical system," IEEE Transactions on Emerging Topics in Computing, vol. 1, no. 1, pp. 148-165, 2013. 
[19] X. Hu, T. H. S. Chu, V. C. M. Leung, and C. H. Ngai, "A survey on mobile social networks: Applications, platforms, system architectures, and future research directions," IEEE Communications Surveys \& Tutorials, vol. 17, no. 3, pp. 15571581, 2014.

[20] C.-L. Fok, M. Hanna, S. Gee et al., "A platform for evaluating autonomous intersection management policies," in Proceedings of IEEE/ACM Third International Conference on Cyber-Physical Systems, pp. 87-96, Beijing, China, April 2012.

[21] S. A. Haque, S. M. Aziz, and M. Rahman, "Review of cyberphysical system in healthcare," International Journal of Distributed Sensor Networks, vol. 2014, Article ID 217415, 2014.

[22] J. Sztipanovits, "Composition of cyber-physical systems," in Proceedings of 14th Annual IEEE International Conference and Workshops on the Engineering of Computer-Based Systems, ECBS 2007, pp. 3-4, usa, March 2007.

[23] E. A. Lee, "Cyber physical systems: design challenges," in Proceedings of the 11th IEEE International Symposium on Object/Component/Service-Oriented Real-Time Distributed Computing (ISORC '08), pp. 363-369, May 2008.

[24] B. Perumal, P. Rajasekaran, and M. H. M. Ramalingam, "Wsn integrated cloud for automated telemedicine (atm) based ehealthcare applications," in Proceedings of International Proceedings of Chemical Biological \& Environmenta, 2012.

[25] X. Hu, J. Zhao, B.-C. Seet, V. C. M. Leung, T. H. S. Chu, and H. Chan, "S-aframe: agent-based multilayer framework with context-aware semantic service for vehicular social networks," IEEE Transactions on Emerging Topics in Computing, vol. 3, no. 1, pp. 44-63, 2015.

[26] X. Hu, X. Li, E. C.-H. Ngai, V. C. M. Leung, and P. Kruchten, "Multidimensional context-aware social network architecture for mobile crowdsensing," IEEE Communications Magazine, vol. 52, no. 6, pp. 78-87, 2014.

[27] L. Zhou, J. Zhang, B. Seet et al., "Software Defined Small Cell Networking under Dynamic Traffic Patterns," in Proceedings of IEEE Cyber Science and Technology Congress, Auckland, New Zealand, August 2016.

[28] A. K. Jain, M. N. Murty, and P. J. Flynn, "Data clustering: a review," ACM Computing Surveys, vol. 31, no. 3, pp. 264-323, 1999.

[29] B. S. Everitt, G. Dunn, B. S. Everitt, and G. Dunn, "Cluster analysis," lem plus 0.5em minus 0.4em Wiley, 2011.

[30] A. K. Jain and R. C. Dubes, "Algorithms for clustering data," Technometrics, vol. 32, no. 2, pp. 227-229, 1988.

[31] E. W. Forgy, "Cluster analysis of multivariate data efficiency vs. interpretability of classification," Biometrics, vol. 21, no. 3, pp. 41-52, 1965.

[32] J. MacQueen, "Some methods for classification and analysis of multivariate observations," in Proceedings of the Berkeley Symposium on Mathematical Statistics and Probability, pp. 281297, University of California Press, Berkeley, California, 1967.

[33] V. Estivill-Castro and J. Yang, "A fast and robust general purpose clustering algorithm," in Proceedings of the Pacific Rim International Conference on Artificial Intelligence, pp. 208-218, Springer-Verlag, London, UK, 1999.

[34] B. Schölkopf, A. Smola, and K.-R. Müller, "Nonlinear component analysis as a kernel eigenvalue problem," Neural Computation, vol. 10, no. 5, pp. 1299-1319, 1998.

[35] U. von Luxburg, "A tutorial on spectral clustering," Statistics and Computing, vol. 17, no. 4, pp. 395-416, 2007.
[36] A. Y. Ng, M. I. Jordan, and Y. Weiss, "On spectral clustering Analysis and an algorithm," in Proceedings of the Advances in Neural Information Processing Systems, vol. 14, pp. 849-856, San Francisco, CA, USA, 2002.

[37] P. J. Rousseeuw, "Silhouettes: a graphical aid to the interpretation and validation of cluster analysis," Journal of Computational and Applied Mathematics, vol. 20, no. 20, pp. 53-65, 1987.

[38] L. Kaufman and P. Rousseeuw, Finding Groups in Data: An Introduction to Cluster Analysis, John Wiley \& Sons, New York, NY, USA, 1990. 

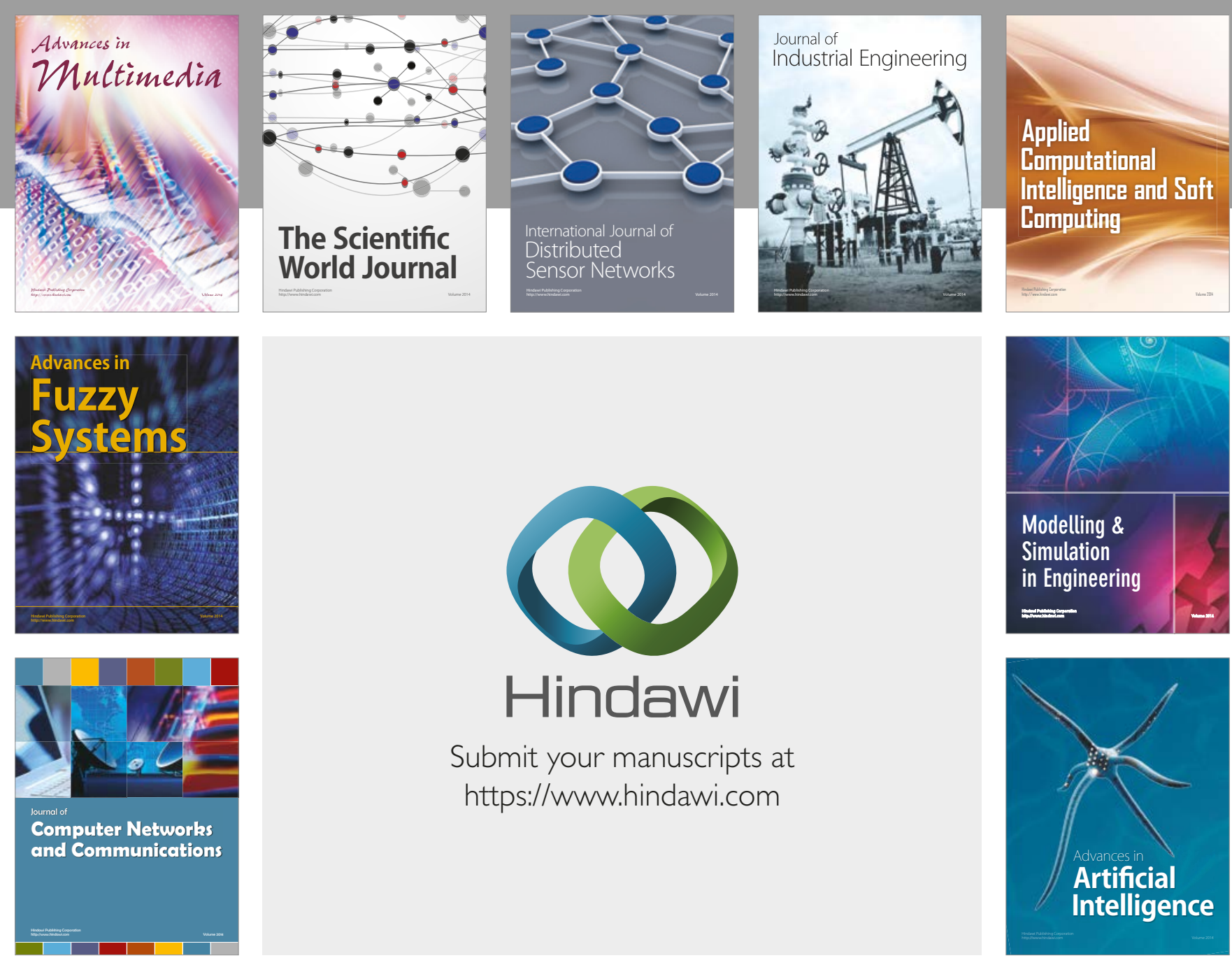

\section{Hindawi}

Submit your manuscripts at

https://www.hindawi.com
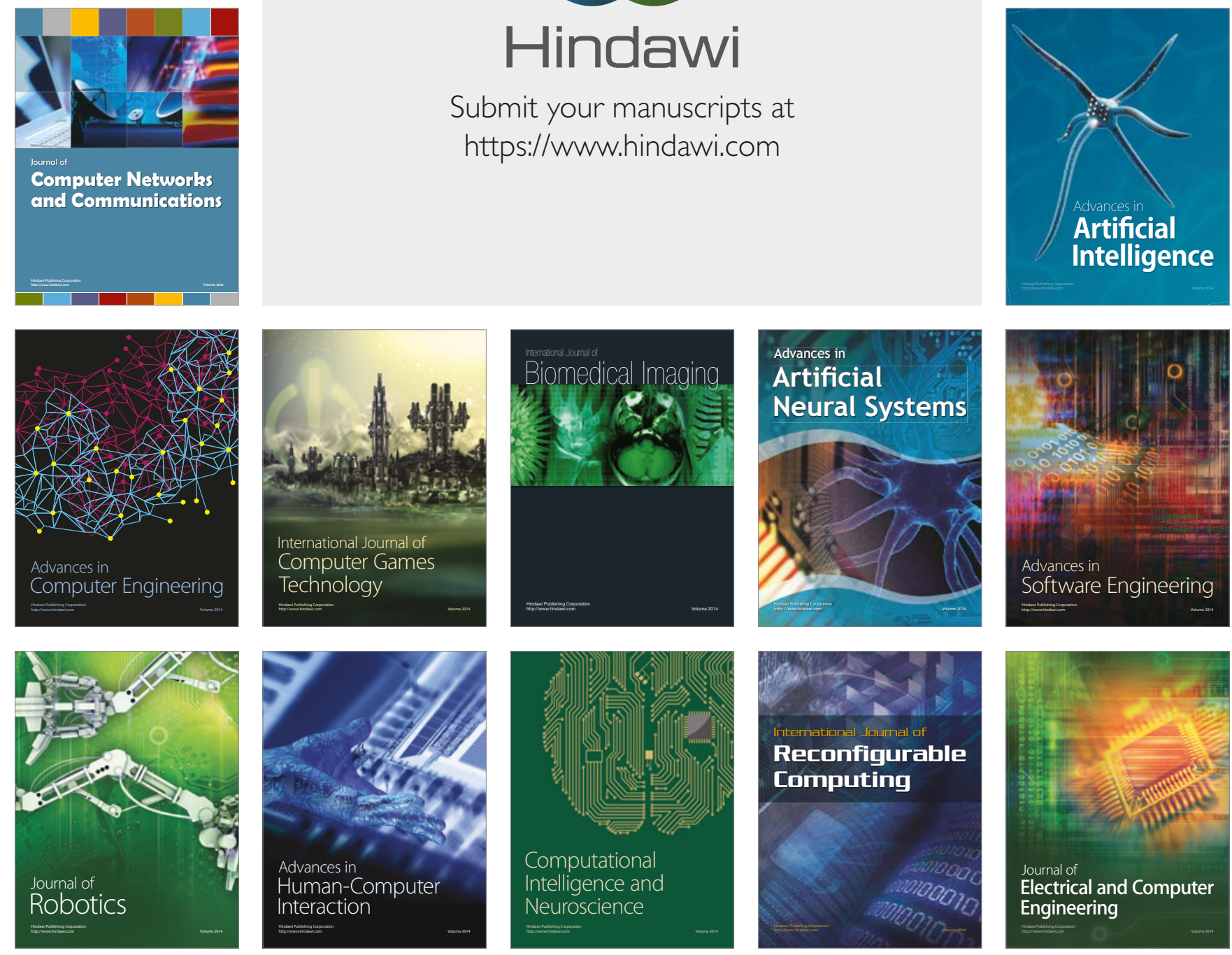